Radu Miculescu, University of North Texas, Department of Mathematics, Denton, Texas, 76203, U.S.A. and University of Bucharest, Department of Mathematics, 14 Academiei Street, 70109, Bucharest, Romania. e-mail: miculesc@netscape.net

\title{
APPROXIMATION OF CONTINUOUS FUNCTIONS BY LIPSCHITZ FUNCTIONS
}

\begin{abstract}
Georganopoulos (see [1] ) has shown that a continuous function $f$ : $X \rightarrow B$, where $X$ is a compact metric space and $B$ a convex subset of a real normed space $Y$, is the uniform limit of a sequence of Lipschitz maps from $X$ to $B$.

In this note we obtain a similar result, namely we show that a continuous function $f: X \rightarrow \mathbb{R}$, where $X$ is a metric space, is a uniform limit of a sequence of locally Lipschitz maps from $X$ to $\mathbb{R}$.

When $X$ is compact and $B=Y=\mathbb{R}$, we get the Georganopoulos' result.
\end{abstract}

\section{Introduction}

Definition 1. A map $f$ of a metric space $(X, d)$ into a metric space $\left(Y, d^{\prime}\right)$ is said to be Lipschitz, if there is a constant $M \geq 0$ such that $\mathrm{d}^{\prime}(f(x), f(y)) \leq$ $M \cdot d(x, y)$ for all $x, y$ in $X$.

If every $x \in X$ has a neighborhood $U$ such that $f_{\mid U}$ is Lipschitz, $f$ is said to be locally Lipschitz (abbreviated LIP).

Definition 2. A LIP-partition of unity, subordinated to an open cover $\left(U_{j}\right)_{j \in J}$ of a metric space $X$, is a family $\left(\varphi_{j}\right)_{j \in J}$ of LIP maps $\varphi_{j}: X \rightarrow[0,1]$ such that:

i) the supports $\operatorname{spt} \varphi_{j}=\overline{\varphi_{j}^{-1}(0,1]}$ form a locally finite family,

ii) $\operatorname{spt} \varphi_{j} \subseteq U_{j}$ for all $j \in J$ and

Key Words: Lipschitz functions, continuous function, approximation

Mathematical Reviews subject classification: 26A16, 54C30 and 41A30

Received by the editors March 10, 2000 
iii) $\sum_{j \in J} \varphi_{j}(x)=1$ for all $x \in X$.

Theorem 1. ( LIP- partition of unity, see [2]) Let $\left(U_{j}\right)_{j \in J}$ be an open cover of a metric space $X$. Then, there is a LIP-partition of unity, subordinated to this cover.

\section{The Result}

Theorem 2. Let $X$ be a metric space. Then, given a continuous function $f: X \rightarrow \mathbb{R}$, there exists a sequence of LIP functions $\left(f_{n}\right)_{n \geq 1}, f_{n}: X \rightarrow \mathbb{R}$, such that $f_{n} \stackrel{u}{\rightarrow} f$

Proof. For each $r \in \mathbb{Q}$, let us consider the set

$$
U_{r}^{1}=\{y \in X \mid f(y)-1<r\} \cap\{y \in X \mid r<f(y)+1\} .
$$

Due to the fact that $f$ is continuous, $U_{r}^{1}$ is open for all $r \in \mathbb{Q}$. As $\left(U_{r}^{1}\right)_{r \in \mathbb{Q}}$ is an open cover of $X$, we can consider, according to Theorem $1,\left(\varphi_{r}^{1}\right)_{r \in \mathbb{Q}}$ a LIPpartition of unity subordinated to this cover. Then, let us consider $f_{1}: X \rightarrow \mathbb{R}$ given by

$$
f_{1}(y)=\sum_{r \in \mathbb{Q}} r \cdot \varphi_{r}^{1}(y) .
$$

It is clear that $f_{1}$ is LIP because $\varphi_{r}^{1}$ are LIP.

For $y \in X$, if $y \in \operatorname{spt} \varphi_{r_{i}}^{1} \subseteq U_{r_{i}}^{1}, i \in\{1, \ldots, n\}$ and $y \notin s p t \varphi_{r}^{1}$ for $r \notin$ $\left\{r_{1}, \ldots, r_{n}\right\}$, then

$$
\begin{aligned}
f(y)-1 & =\sum_{i=1}^{n} \varphi_{r_{i}}^{1}(y) \cdot(f(y)-1)<\sum_{i=1}^{n} r_{i} \cdot \varphi_{r_{i}}^{1}(y) \\
& =f_{1}(y)<\sum_{i=1}^{n} \varphi_{r_{i}}^{1}(y) \cdot(f(y)+1)=f(y)+1,
\end{aligned}
$$

i.e.

$$
f(y)-1<f_{1}(y)<f(y)+1
$$

for all $y \in X$.

Now, for each $r \in \mathbb{Q}$, let us consider the set

$$
\begin{gathered}
U_{r}^{2}=\left\{y \in X \mid \max \left\{f(y)-\frac{1}{2}, f_{1}(y)-\frac{1}{2}\right\}<r\right\} \cap \\
\cap\left\{y \in X \mid r<\min \left\{f(y)+\frac{1}{2}, f_{1}(y)+\frac{1}{2}\right\} .\right.
\end{gathered}
$$

Due to the fact that $f(y)-\frac{1}{2}, f_{1}(y)-\frac{1}{2}, f(y)+\frac{1}{2}$ and $f_{1}(y)+\frac{1}{2}$ are continuous, $U_{r}^{2}$ is open for all $r \in \mathbb{Q}$. As $\left(U_{r}^{2}\right)_{r \in \mathbb{Q}}$ is an open cover of $X$, 
we can consider, according to Theorem 1, $\left(\varphi_{r}^{2}\right)_{r \in \mathbb{Q}}$ a LIP-partition of unity subordinated to this cover. Let us consider $f_{2}: X \rightarrow \mathbb{R}$ given by

$$
f_{2}(y)=\sum_{r \in \mathbb{Q}} r \cdot \varphi_{r}^{2}(y) .
$$

Exactly as above we have that $f_{2}$ is LIP and

$$
\max \left\{f(y)-\frac{1}{2}, f_{1}(y)-\frac{1}{2}\right\}<f_{2}(y)<\min \left\{f(y)+\frac{1}{2}, f_{1}(y)+\frac{1}{2}\right\} .
$$

Continuing these process we obtain for each $n \in N$ a LIP function $f_{n}: X \rightarrow \mathbb{R}$ such that

$$
\begin{aligned}
\max \{ & \left.f(y)-\frac{1}{2^{n-1}}, f_{n-1}(y)-\frac{1}{2^{n-1}}\right\}<f_{n}(y)< \\
& <\min \left\{f(y)+\frac{1}{2^{n-1}}, f_{n-1}(y)+\frac{1}{2^{n-1}}\right\} .
\end{aligned}
$$

It is clear that

$$
\left|f_{n}(y)-f_{n-1}(y)\right|<\frac{1}{2^{n-1}} .
$$

Consequently, for each $n, p \in \mathbb{N}$ and for each $y \in X$ we have

$$
\left|f_{n+p}(y)-f_{n}(y)\right|<\frac{1}{2^{n-1}} \cdot\left(1-\frac{1}{2^{p}}\right)<\frac{1}{2^{n-1}} .
$$

Hence $\left(f_{n}(y)\right)_{n \in \mathbb{N}}$ is uniformly Cauchy, so $\left(f_{n}(y)\right)_{n \in \mathbb{N}}$ is uniformly convergent. Moreover for each $n \in \mathbb{N}$ and for each $y \in X$ we have

$$
f(y)-\frac{1}{2^{n-1}}<f_{n}(y)<f(y)+\frac{1}{2^{n-1}} .
$$

This implies that $\lim _{n \rightarrow \infty} f_{n}(y)=f(y)$ uniformly.

Remark. There exists continuous functions that cannot be the uniform limit of a sequence of Lipschitz functions. So, in this sense, our result is the best one can obtain.

For example, the continuous function $f: \mathbb{R} \rightarrow \mathbb{R}, f(x)=e^{x}$, has the above property. Indeed, let us suppose that there exists a sequence of Lipschitz functions $f_{n}: \mathbb{R} \rightarrow \mathbb{R}$ such that $f_{n} \rightarrow f$ uniformly. Then there exists an integer $n_{0}$ so that

$$
\left|f_{n_{0}}(x)-f(x)\right|<\frac{1}{2} \text { for every } x \in \mathbb{R} .
$$

Since $f_{n_{0}}$ is Lipschitz there exists a constant $M_{n_{0}}$ such that

$$
\left|f_{n_{0}}(x)-f_{n_{0}}(y)\right|<M_{n_{0}}|x-y| \text { for every } x, y \in \mathbb{R} .
$$


Hence, for every $x, y \in \mathbb{R}$, we have

$$
|f(x)-f(y)| \leq\left|f_{n_{0}}(x)-f(x)\right|+\left|f_{n_{0}}(x)-f_{n_{0}}(y)\right|+\left|f_{n_{0}}(y)-f(y)\right|
$$

so, we obtain

$$
|f(x)-f(y)|<1+M_{n_{0}}|x-y| .
$$

According to the mean value theorem, for every $x, y \in \mathbb{R}$, there exists $\zeta$ between $x$ and $y$ such that

$$
f(x)-f(y)=f^{\prime}(\zeta)(x-y) .
$$

Hence

$$
|x-y|\left(e^{\zeta}-M_{n_{0}}\right)<1 .
$$

Choosing $y=n+1$ and $x=n$, where $n$ is an arbitrary integer, we have

$$
e^{n}-M_{n_{0}}<1 \text { for each } n \in \mathbb{N} \text {. }
$$

The last relation obviously is not true.

\section{References}

[1] G. Georganopoulos, Sur l'approximation des fonctions continues par des fonctions lipschitziennes, C. R. Acad. Sci. Paris, Ser A, 264(7) (1967), 319-321.

[2] J. Luukainnen and J. Vaisala, Elements of Lipschitz Topology, Ann. Acad. Sci. Fenn. Ser. A.I., Math. 3(1977), 85-122. 